\title{
Drop impact on natural porous stones
}

\author{
J.B. Lee ${ }^{1}$, D.Derome ${ }^{2} \&$ J.Carmeliet $^{1,2^{*}}$
}

${ }^{1}$ Chair of Building Physics, ETH Zurich, Stefano-Franscini-Platz 5, CH-8093 Zürich, Switzerland,

${ }^{2}$ Laboratory for Multiscale studies in Building Physics, Swiss Federal Laboratories for Materials

Science and Technology, EMPA, Überlandstrasse 129, CH-8600 Dübendorf, Switzerland

\section{Abstract}

Drop impact and spreading on three natural porous stones is experimentally determined using highspeed imaging and compared with spreading over an impermeable steel surface. The dynamic nonwetting behavior during spreading and the hydrophobic contact angle $>90^{\circ}$ is attributed to the presence of an air layer between droplet and porous substrate. As the contact line pins at maximum spreading on porous stones, the maximum spreading determines the liquid contact area on such substrate. The droplet gets pinned when the air layer is broken at the contact line and capillary forces develop in fines pores at the droplet edge pinning the droplet. Maximum spreading on porous stones increases with impact velocity but does not scale with Weber number at low impact velocity. It is demonstrated that dynamic wetting plays an important role in the spreading at low velocity and that the dynamic wetting as characterized by the dynamic contact angle $\theta_{\mathrm{D}}$ has to be taken into account for predicting the maximum spreading. Correcting the maximum spreading ratio for the dynamic wetting behavior, it is demonstrated that all data for porous stones and non-porous substrates collapse onto a single curve.

\section{Introduction}

The study of impacting drops spreading over porous media has received little attention compared to similar studies on impermeable surfaces, although such event is more commonplace and often plays an important role in several processes [1]. One of the first studies for drop impact on permeable surface was performed by Wallace and Yoshida [2]. They investigated the spread factor defined as the ratio of the diameter of a stain to the initial drop diameter on paper, as a function of impact energy for pesticide spray application. Chandra and Avedisian [3] compared drop impact on impermeable stainless steel surfaces and porous ceramic surfaces. They used the difference of volume before and after impact to estimate the volume absorbed into the ceramic substrate. Although such difference was measured to be between 12 to $15 \%$, considering the time scale of maximum spreading (under $5 \mathrm{~ms}$ ), the absorbed volume was finally neglected in their maximum spreading prediction. Previous work 
focused mainly on the depletion of the droplet from the surface and on the influence of properties of porous media (porosity, pore size) and liquids on the impact and depletion processes.

Less information is available about liquid (re)distribution inside the porous media after drop impact.In terms of computational work, Zhao et al. [4] and Reis et al. [5,6] performed numerical simulations of drop impact on porous media with volume-of-fluid method. Zhao et al. [4] compared the experimental results of the drop impact on heated porous media with simulation results. They did not consider absorption given the presence of a vapor layer on the porous substrate. Reis et al. $[5,6]$ developed a numerical model to resolve both the shape of the impacting drop on the surface and the liquid content distribution in the porous medium. They performed a parametric study for impact condition, permeability, porosity, pore size and wettability and found the simulated droplet shape to agree with what is seen in experiments. The predicted liquid content in the porous medium compared well with magnetic resonance imaging results after full absorption. The shape of the droplet in the porous medium is similar to a half-spheroid, which aspect ratio depends on the porous medium and liquid droplet characteristics [5].

More recently, drop impact on granular media has been quite investigated. Marston et al. [7] studied the maximum droplet spreading diameter on packed glass beads for different liquids, i.e. $50 \%$ ethanol-50\% water, ethanol, water and acetone, and reported that the maximum spreading scales with $\mathrm{We}^{1 / 5}$. Katsuragi [8] studied the size of craters in granular layers resulting from water drop impact. He assumed that the crater size is determined by the maximum spreading diameter of droplet and reported that the crater size scales with $\mathrm{We}^{1 / 4}$, the same scaling proposed by Clanet et al. [9] for smooth impermeable (no deformable) substrates. Delon et al. [10] also reported a $D_{\max } / D_{0} \sim \mathrm{We}^{1 / 4}$ scaling when studying water drop impact on sand substrates of different grain sizes. Nefzaoui and Skurtys [11] reported that maximum droplet spreading on dry glass bead substrate scales with $\mathrm{We}^{1 / 5}$ for water droplet and with $\mathrm{We}^{1 / 4}$ for liquids with higher surface tension. Zhao et al. [12] showed that crater size scales with impact energy on substrates of different grain sizes. Going further, Zhao et al. [13] showed that maximum spreading for water drop impacting dry glass beads granular media scales with the effective Weber number defined by the maximum crater depth.

For drop impact on porous media, the influence of the porous medium on spreading is still insufficiently understood due to the presence of simultaneous behaviors, spreading and absorption, and due to the lack of knowledge of the contact line behavior on porous and rough surfaces. There is a clear need to quantify properly droplet spreading on porous media. In this paper, we determine experimentally the drop impact and spreading on three natural porous stones using high-speed imaging and compare it with the behavior on an impermeable surface. Especially we analyze maximum spreading at low impact velocity in view of the dynamic wetting behavior as characterized by the dynamic contact angle. We finally propose a method to scale all data into a single curve taking into account the dynamic contact angle. 


\section{Methods and Material}

Three natural stones are selected for droplet impact experiments on porous media: Savonnières, Meule and Pietra Serena. Savonnières is highly porous and a quasi-pure calcitic stone $(99.8 \%$ $\mathrm{CaCO}_{3}$ ), and is used as a building material on facades of historical buildings (e.g. railway station Gare de l'Est in Paris), as a stone for sculptures (e.g. the sculptures on the facade of the cathedrals in Aachen and Cologne), and is applied for restoration purposes [14,15]. The sandstone Meule (grès à meules) is composed of quartz grain (74\%), with clay and other secondary mineralization, and is used as a building material (e.g. tower of the cathedral of Strasbourg) [16]. Pietra Serena is a fine-grained and compact sandstone, and is used widely as a building material for columns, cornices and arches and as a stone for sculptures (e.g. Pazzi and Medici chapels in Florence). Figure 1 shows microscope images of the porous stones. The porous stone samples are prepared by cutting cubes $(20 \times 20 \times 20$ $\mathrm{mm}^{3}$ ). The bulk density $\rho_{\text {bulk }}$ defined as the ratio of the dry mass to the total volume, the open porosity $\Phi$ defined as the ratio of the volume of open pores to the total volume and the saturated water content $w_{\text {sat }}$ defined as the mass of water filling the open pores per total volume are measured. The water absorption coefficient $A_{\text {cap }}$ is determined by measuring the absorption mass rate per unit surface in a free water uptake experiment. The capillary water content $w_{\text {cap }}$ equals the water content when the water front reaches the top of the sample during capillary absorption. The impervious surface is steel, with an arithmetic average roughness of $0.42 \mu$ meters and an equilibrium contact angle of $\theta_{\mathrm{eq}}=61^{\circ}$.

In Table 1, the measured properties of the three stones are summarized. The stones are selected mainly for their range of open porosity in order to understand the influence of surface pore structure on the dynamics of droplet spreading. Elaborate studies on the pore structure, moisture and mechanical properties of Savonnières and Meule can be found in $[14,16,17]$. Savonnières shows the highest porosity, a faster water absorption rate and the largest capillary water content compared to the other stones. The capillary water content is smaller than the saturated water content due to air entrapment during imbibition from a free water surface. Pietra Serena shows the lowest porosity, water uptake coefficient and capillary water content. The portion of the pore space filled by water at capillary moisture content in Savonnières, Meule and Pietra Serena is respectively $56 \%, 72 \%$ and $80 \%$.

The arithmetic average roughness $R_{a}$ for Savonnières, Meule and Pietra serena is respectively 10, 9 and $4 \mu \mathrm{m}$, showing the porous materials are quite rough due to the sawing process, compared to the smooth steel surface. The equivalent pore radius $R_{e q}$, defined from pore size distribution measurement using mercury intrusion porosimetry (MIP) $[14,16]$ and scanning electron microscope (SEM) image [18], for Savonnières, Meule and Pietra Serena equals respectively 100, 10 and $0.04 \mu \mathrm{m}$. It was not possible to measure the equilibrium contact angle on stones due to immediate spreading of the droplet due capillary uptake by the substrate, so no equilibrium could be found. The main components of the 
stones display very low contact angles (calcite $\sim 0^{\circ}$ and quartz $\sim 11$ to $19^{\circ}$ ) showing they are almost perfectly wetting [19-21].

(a)

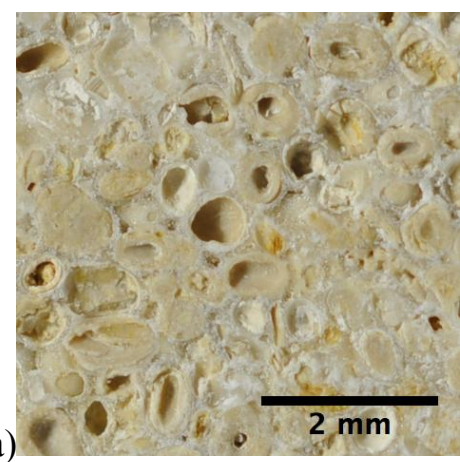

(b)

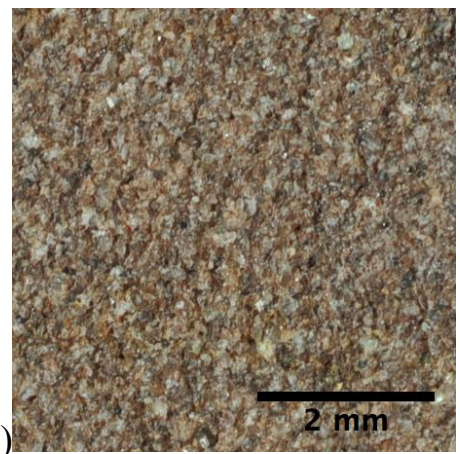

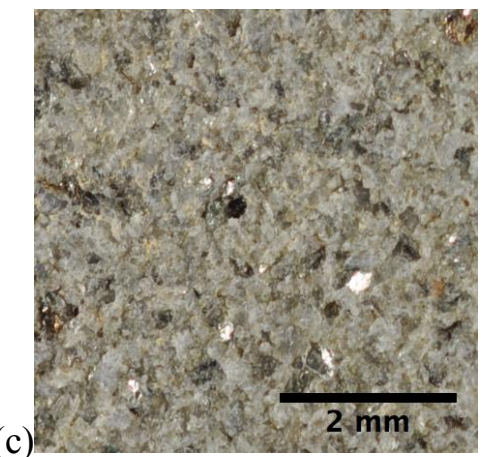

Fig. 1 Microscope images of porous stones: (a) Savonnières, (b) Meule and (c) Pietra Serena.

Table 1 Properties of porous stones

\begin{tabular}{lrrr}
\hline & Savonnières & Meule & Pietra Serena \\
\hline$\rho_{\text {bulk }}\left(\mathrm{kg} / \mathrm{m}^{3}\right)$ & $1974.5 \pm 38.7$ & $2253.2 \pm 14.3$ & $2558.7 \pm 21.9$ \\
$\Phi(\%)$ & $26.9 \pm 1.4$ & $16.6 \pm 0.3$ & $5.1 \pm 0.6$ \\
$w_{\text {sat }}\left(\mathrm{kg} / \mathrm{m}^{3}\right)$ & $268.2 \pm 13.6$ & $165.7 \pm 4.0$ & $50.9 \pm 6.0$ \\
$w_{\text {cap }}\left(\mathrm{kg} / \mathrm{m}^{3}\right)$ & $151.1 \pm 6.4$ & $119.8 \pm 4.0$ & $41.6 \pm 2.5$ \\
$A_{\text {cap }}\left(\mathrm{kg} / \mathrm{m}^{2} \mathrm{~s}^{1 / 2}\right)$ & $0.089 \pm 0.012$ & $0.028 \pm 0.003$ & $0.004 \pm 0.001$ \\
$R_{a}(\mu \mathrm{m})$ & $10.3 \pm 3.5$ & $9.1 \pm 1.5$ & $4.4 \pm 1.5$ \\
$R_{e q}(\mu \mathrm{m})$ & 100 & 10 & 0.04 \\
\hline
\end{tabular}

Water drop impact on porous stone is recorded in shadowgraphy using a high-speed camera (10 000 frame per second, $7.38 \mu \mathrm{m}$ spatial resolution and $5 \mu$ s exposure time). The properties of water and the impact conditions are given in Table 2. The drop impact test is repeated more than 10 times with 3 different samples at arbitrary surface locations for each measuring condition in order to obtain sufficient reproducibility. Experimental results are reported by their average value (symbol) and standard deviation (error bar). Drop impact on porous stones is compared with the drop impact on impermeable steel surface. Images captured from high-speed camera are analyzed with a custom made image analysis MATLAB code for determination of: the initial droplet diameter $D_{0}$, the impact velocity $V_{\mathrm{i}}$, the spreading diameter at the rim $D(t)$, the spreading diameter at the contact line $D_{\mathrm{CL}}(t)$, the dynamic contact angle $\theta_{\mathrm{D}}$, the maximum spreading ratio $\beta_{\max }=D_{\max } / D_{0}$ and the time at maximum spreading $t_{\max }$. The inset in figure 2 shows the difference between $D$ and $D_{\mathrm{CL}}$ during the evolution of spreading. The dynamic contact angle is obtained from the image by applying a goniometric mask 
developed in [22] on a region of $100 \mu \mathrm{m}$ vertically above the surface line as also applied in [23]. This method was found to give good a compromise between accuracy and level of noise and blurring at contact line and to yield a good reproducibility.

To determine the mass of water in the porous stone $M_{\mathrm{abs}}$ due to inertial effects at impact or due to capillary absorption, the drop volume remaining on the surface is estimated assuming the shape of a cap method and subtracted from the initial mass of the droplet $M_{0}$. The volume $V_{\text {drop }}$ of a drop on a surface having a spherical cap is given by:

$$
V_{\text {drop }}(t)=\frac{\pi h(t)}{6}\left(3\left(\frac{D_{C L}(t)}{2}\right)^{2}+h(t)^{2}\right)
$$

where $h$ is the height of the spherical cap. We found that the evaporation of a drop deposited on a nonporous surface is negligible during the time of investigation $(\Delta t \sim 0.01 \mathrm{~s})$, as the volume loss in 10 seconds is less than $3 \%$. We also evaluated the accuracy of the spherical cap approach for determining the remaining volume $V_{\text {drop }}$ by depositing a droplet on an impermeable surface. The global systematic error was found to be less than $3 \%$. The mass in the stone $M_{\text {pore }}$ is then given by:

$$
M_{\mathrm{abs}}=M_{0}-\rho \cdot V_{\mathrm{drop}}
$$

Table 2 Properties of water droplet and impact conditions for drop test

\begin{tabular}{cccccccc}
\hline & \multicolumn{3}{c}{ Properties at $25^{\circ} \mathrm{C}$} & \multicolumn{4}{c}{ Impact conditions } \\
& $\begin{array}{c}\rho \\
\left(\mathrm{kg} / \mathrm{m}^{3}\right)\end{array}$ & $\begin{array}{c}\mu \\
(\mathrm{mPa} \cdot \mathrm{s})\end{array}$ & $\begin{array}{c}\gamma \\
(\mathrm{mN} / \mathrm{m})\end{array}$ & $\begin{array}{c}D_{0} \\
(\mathrm{~mm})\end{array}$ & $\begin{array}{c}V_{\mathrm{i}} \\
(\mathrm{m} / \mathrm{s})\end{array}$ & We & Re \\
\hline water & 998 & 1.0 & 72.8 & 2.0 & $0.2-4.0$ & $1-440$ & $400-8000$ \\
\hline
\end{tabular}

\section{Spreading and deposition}

The time evolution of the spreading diameters at rim $D(\mathrm{t})$ and at contact line $D_{\mathrm{CL}}(\mathrm{t})$ after impact for drop impacting on Savonnières is presented in Figure 2a. Figure $2 b$ shows snapshots at maximum spreading for the different impact velocities. The spreading diameter at rim and the contact line increase with impact velocity due to higher initial kinetic energy of the drop. The difference between spreading diameter at rim and contact line is rather small, especially at maximum spreading. In the following, we use the contact line diameter to characterize the spreading of the drop during impact on the porous substrate. 


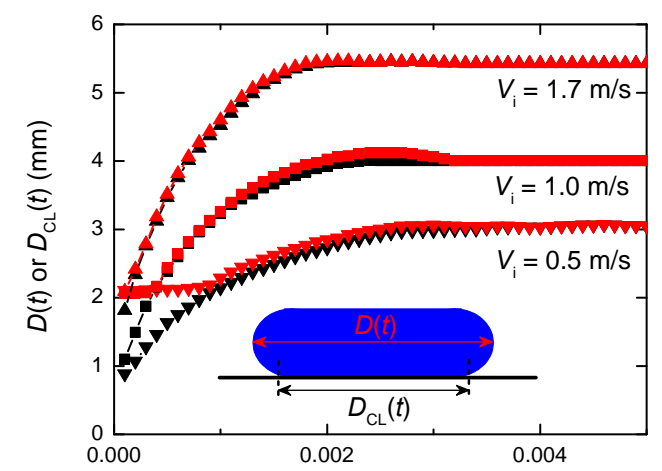

(a)

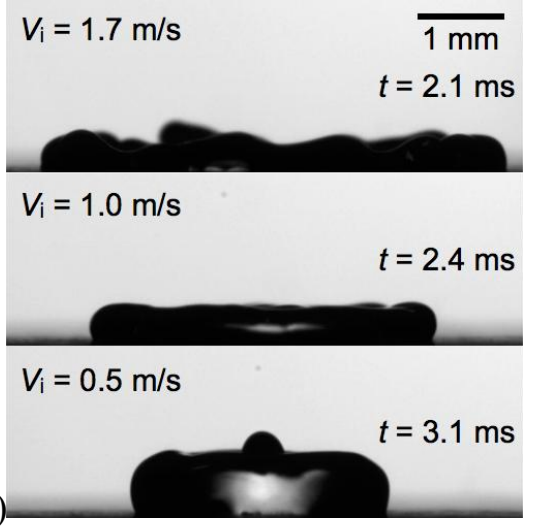

(b)

Figure 2: (a) Time evolution of spreading diameter at rim $D$ (red) and at contact line $D_{\mathrm{CL}}$ (black) and (b) snapshots at maximum spreading for different impact velocities on Savonnières limestone.

We select the impact velocity at $V_{\mathrm{i}}=1.0 \mathrm{~m} / \mathrm{s}$ to compare the spreading on the same porous stone and an impermeable steel surface in Fig. 3. In Fig. 3a, we observe that the contact line diameter for both cases first increases until reaching its maximum spreading. During this spreading phase, the dynamic contact angle is higher than $90^{\circ}$, showing a dynamic non-wetting behavior. For the steel surface after reaching the maximum spreading diameter, the contact line diameter reduces during the receding phase (Fig. 3a), characterized by a reduction of the contact angle below $90^{\circ}$ until reaching the equilibrium contact angle indicated by the horizontal red dashed line in Fig. 3b. We see that the contact angle shows some oscillations, when reaching this equilibrium. In contrast, the contact line diameter of Savonnières remains constant indicating the droplet is pinned. While the contact line diameter remains constant, the contact angle decreases showing some oscillations. The inset figures show the drop shape at two moments around an important change of contact angle, while the drop stays pinned.

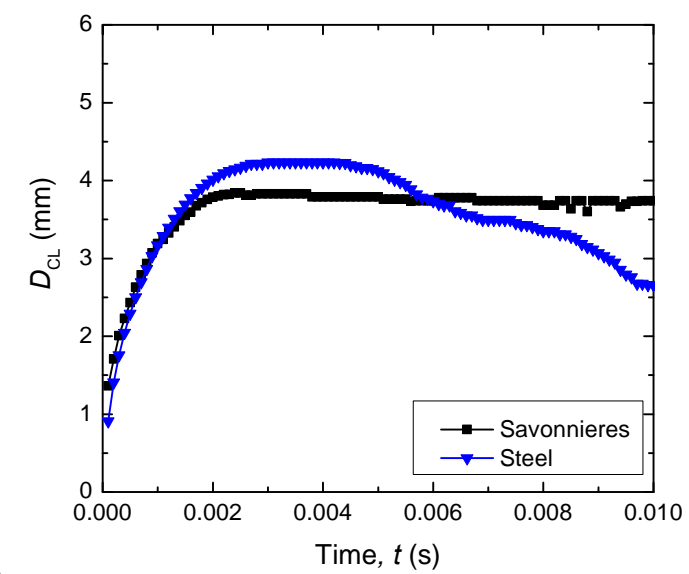

(a)

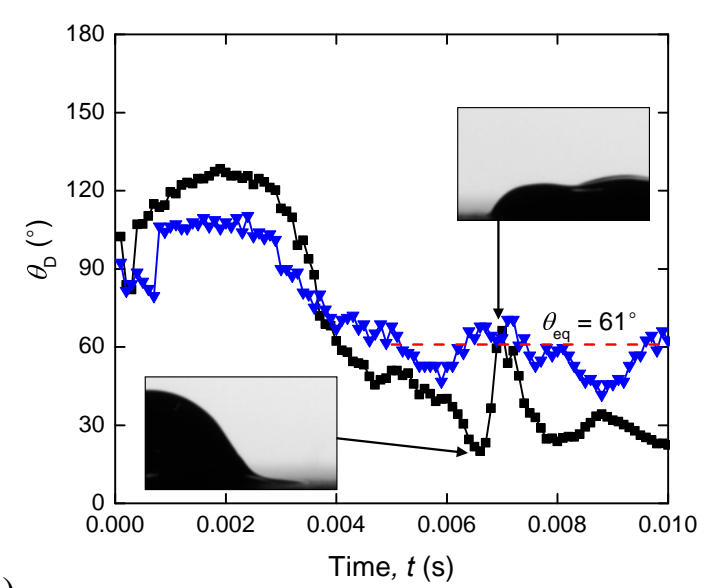

(b)

Figure 3: Comparison of drop impact on porous Savonnières and impermeable steel: (a) spreading diameter at contact line $D_{\mathrm{CL}}$ and (b) dynamic contact angle $\theta_{\mathrm{D}}$. 
Figure 4 shows the comparison of drop impact behavior for the three different porous stones. For all porous stones, the contact line diameter remains constant after reaching its maximum, showing that droplets on all porous surfaces are pinned (Fig. 4a). Droplets on Savonnières and Meule show quite similar evolution of the contact line diameter, while, on Pietra Serena, the droplet spreads more compared with droplets on Savonnières and Meule stones. The dynamic contact angle on the different porous stones shows similar behavior (Fig. 4b): it reaches a maximum during the spreading phase, followed by a decrease of contact angle while staying pinned, followed by some oscillations when reaching its equilibrium value. We observe thus at short time scale a constant contact radius (CCR) mode. The maximum dynamic contact is different for the three porous materials: Savonnières shows the highest contact angle $\theta_{\mathrm{D}} \sim 123^{\circ}$, Meule reaches a contact angle of $\theta_{\mathrm{D}} \sim 108^{\circ}$ and Pietra Serena shows the lowest contact angle $\theta_{\mathrm{D}} \sim 101^{\circ}$. We found that the maximum dynamic contact angle decreases with decreasing volumetric porosity of the porous material: Savonnières $\Phi=27 \%$, Meule $\Phi=17 \%$ and Pietra Serena $\Phi=5 \%$. Assuming the porous media to have a random structure, the areal or surface porosity and volumetric porosity are equal [24]. For Savonnières, an oolithic material with complex pore structure, the ratio between areal and volumetric porosities was found to be 0.87 [18]. This means we find a direct relation between dynamic contact angle and surface porosity.

The dynamic contact angle during spreading shows a dynamic non-wetting or hydrophobic behavior $\left(\theta_{\mathrm{D}}>90^{\circ}\right)$, although the porous stones in quasi-static conditions are clearly hydrophilic as indicated by their capillary active behavior. From the dynamic hydrophobic behavior during spreading and the hydrophilicity seen in equilibrium conditions, we infer that, at the interface during spreading, air is entrapped in the surface pore structure forming a thin air layer between the liquid and the solid material (Fig. 5a). The air layer promotes the non-wetting behavior and a dynamic contact angle $>90^{\circ}$ during spreading.

Figure $4 \mathrm{c}$ shows a time sequence of snapshots for drop impact on porous stones and steel surface. The droplets on porous stones are pinned at maximum spreading as indicated by the vertical dotted lines. In contrast, the spreading diameter on steel surface reduces until reaching its equilibrium state, showing no pinning occurs on steel.

From the dynamic hydrophobic behavior during spreading and the hydrophilicity seen in equilibrium conditions, we infer that, at the interface during spreading, air is entrapped in the surface pore structure forming a thin air layer between the liquid and the solid material (Fig. 5a). The air layer promotes the non-wetting behavior and a dynamic contact angle $>90^{\circ}$ during spreading.

The pinning on porous stones and no-pinning behavior on steel could be attributed to the difference in roughness between porous stones ( $R_{a}$ from 10 to $4 \mu \mathrm{m}$,) and steel $\left(R_{a} 0.4 \mu \mathrm{m}\right)$. We also performed drop impact measurements on rough sandpaper (Silicon Carbide Paper, BUEHLER) with different grit sizes (P120, P240, P600 and P2500), showing a roughness $R_{a}$ varying between 21 and $3 \mu \mathrm{m}$, to study the influence of roughness on pinning behavior [24]. In all these experiments, we did not 
observe pinning behavior, but a receding of the droplet after maximum spreading. This makes us conclude that the open capillary active pore structure plays an essential role in the pinning and not the roughness. Fig. $5 \mathrm{~b}$ shows a schematic of the pinning process due to capillary absorption at the contact line of the droplet at maximum spreading. Since the contact line velocity decreases when reaching maximum spreading, the air layer between droplet and surface can be broken, leading to capillary contact between droplet and porous substrate. This capillary contact induces capillary forces on the droplet by the small pores showing high capillary suction. The droplet remains pinned at the contact line at maximum spreading due to these high capillary forces in the fines pores at the droplet edge leading also to a wetting behavior and a contact angle $<90^{\circ}$. Inwards of the pinned contact line, air remains entrapped under the droplet. The presence of this air layer has been observed by high-speed camera for Savonnières, demonstrating the adequacy of our assumption on the presence of an air layer (Figure 5c, Movie in the Supplementary Material).

(a)

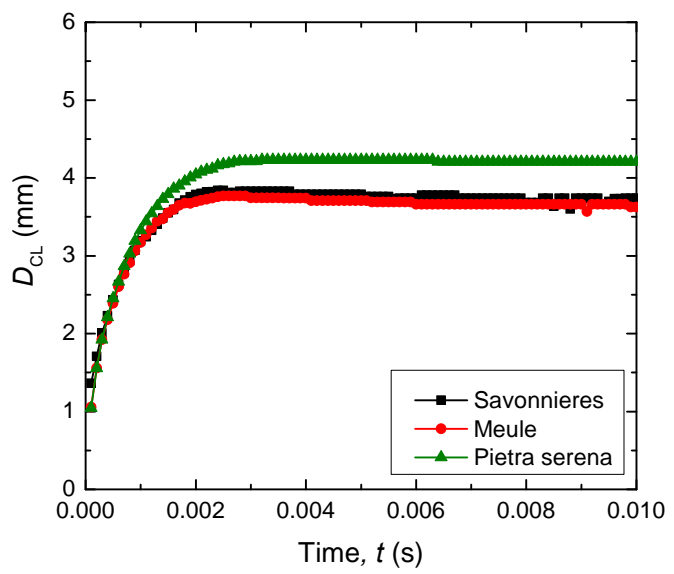

(b)
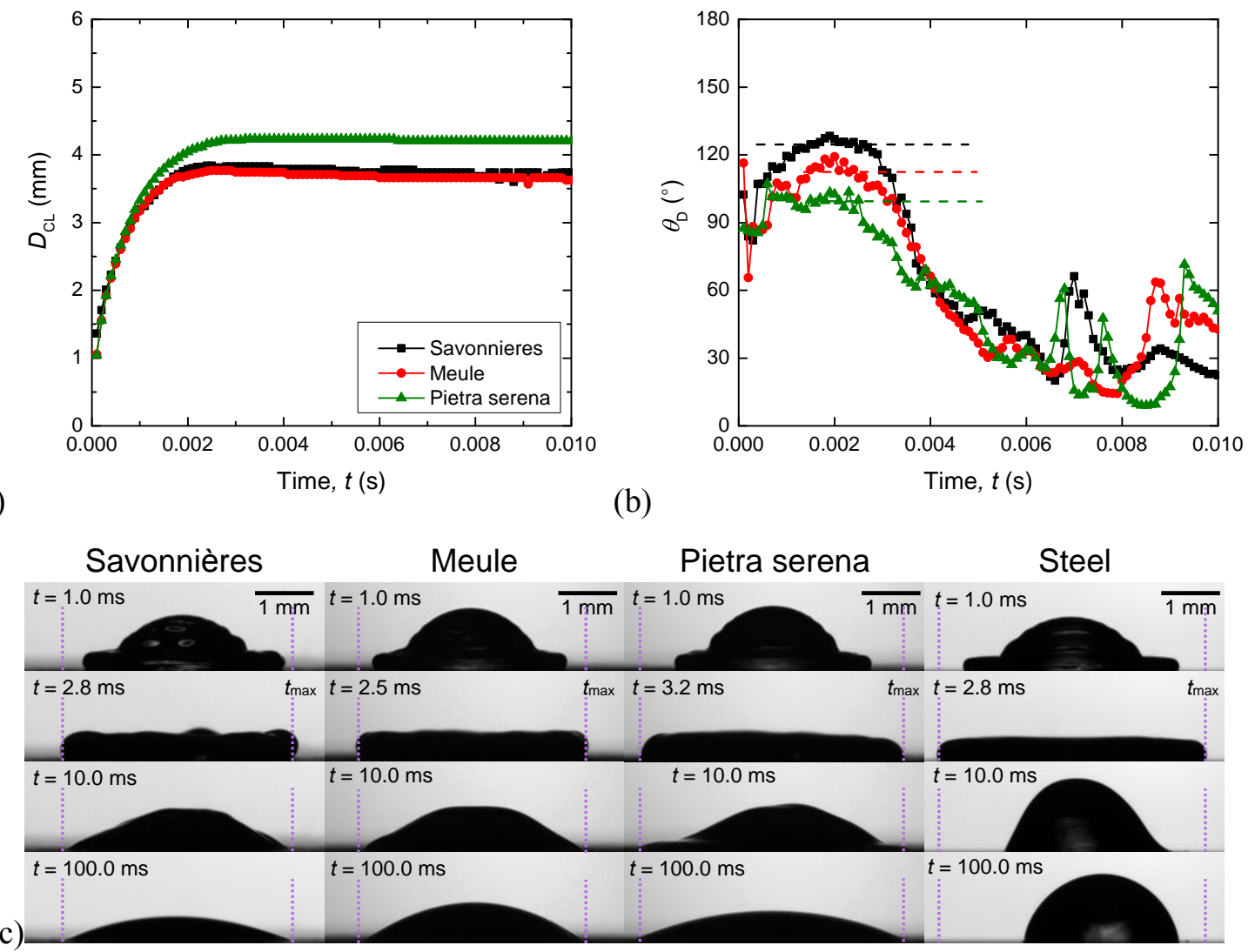

Figure 4: Comparison of drop impacts for $D_{0}=2.0 \mathrm{~mm}$ and $V_{\mathrm{i}} \sim 1.0 \mathrm{~m} / \mathrm{s}$ on different porous stones, (black) Savonnières, (red) Meule and (green) Pietra Serena. (a) Time evolution of spreading diameter at contact line $D_{\mathrm{CL}}$. (b) Dynamic contact angle $\theta_{\mathrm{D}}$ (c) Snapshots of drop impact on porous materials and steel surface. Vertical line indicates contact line diameter at maximum spreading. 
(a) spreading phase
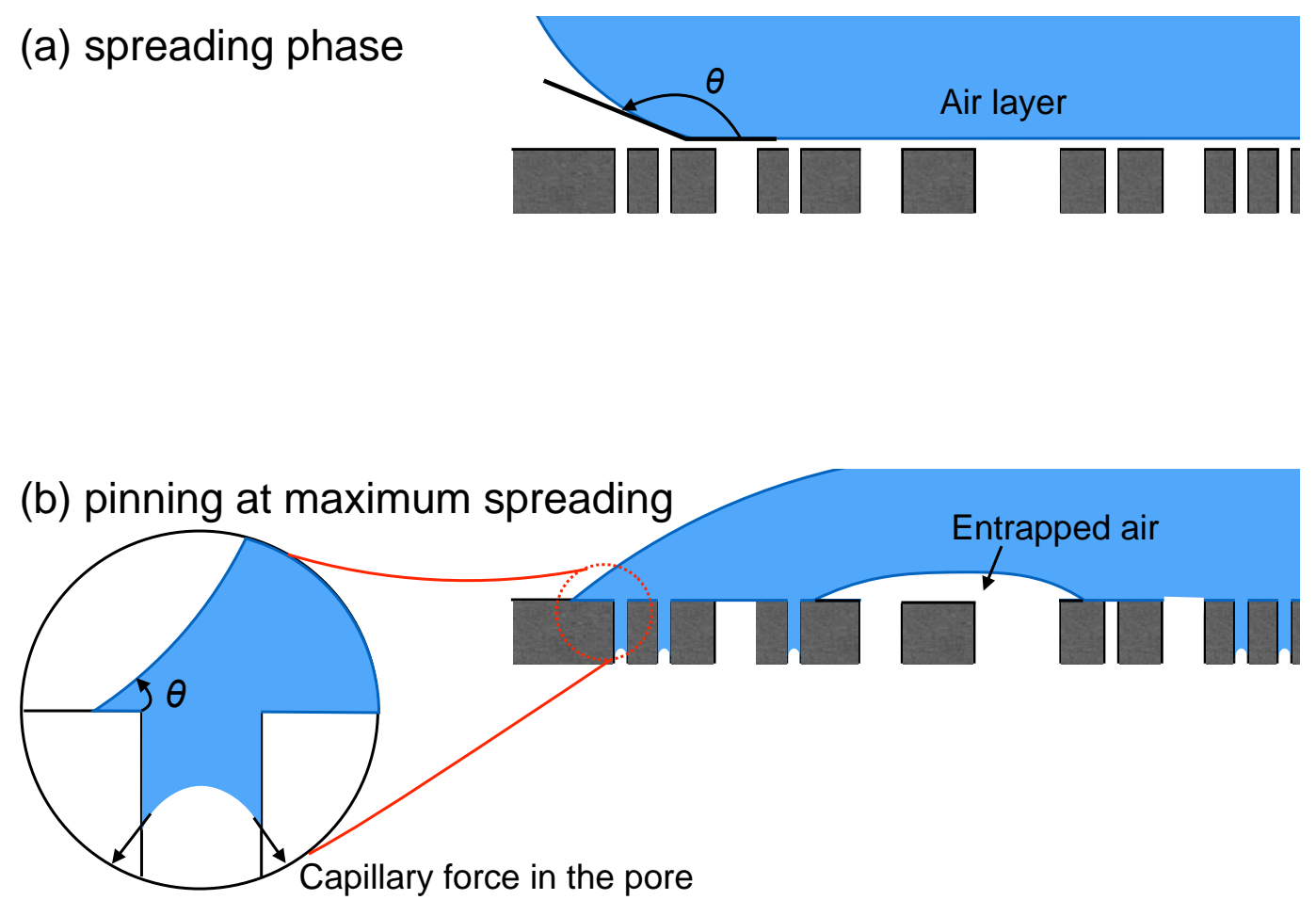

(c) figures of entrapped air

$1 \mathrm{~mm}$

$t=5 \mathrm{~ms}$

$t=6.1 \mathrm{~ms}$

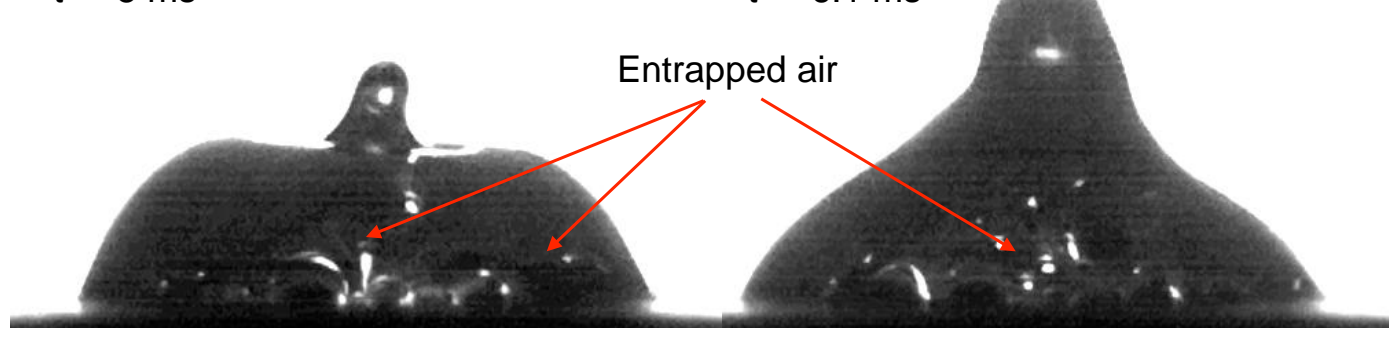

Figure 5: Schematic of dynamic spreading and pinning at maximum spreading. (a) Presence of an air layer between droplet and porous substrate leads to a non-wetting dynamic spreading characterized by a contact angle $>90^{\circ}$. (b) The droplet is pinned at the contact line at maximum spreading due to capillary forces in fines pores at the droplet edge leading to a wetting behavior and a contact angle $<$ $90^{\circ}$. (c) Air remains entrapped under the pinned droplet as shown in receding phase at $t=5$ and 6.1 ms for Savonnières.

\section{Maximum spreading}

The maximum spreading is characterized by the maximum spreading ratio $\beta_{\max }=D_{\max } / D_{0}$. Figure 6 compares the maximum spreading ratio as a function of impact velocity for the three porous stones and impermeable steel surface. The maximum spreading ratio is quite similar for all substrates at high impact velocity. Maximum spreading is determined by both viscosity and surface tension in this region. However, at low impact velocity, the curves start to diverge more and more and finally tend to level off to a different maximum spreading ratio at zero velocity, which will be referred to as $\beta_{\mathrm{Vi}=0}$. 
The value for $\beta_{\mathrm{V} i=0}$ is different for the different substrates and its determination will be discussed below. The maximum spreading ratio at low impact velocity is smaller for Savonnières and Meule compared to the one of impermeable steel surface, while the maximum spreading ratio on Pietra Serena is higher. The lower maximum spreading on a porous medium compared to a solid surface can be explained by the following phenomena: (1) a part of the drop volume penetrates into the porous stone, resulting in less volume available for spreading and a decrease of the spreading; (2) the dynamic wetting behavior influences the maximum spreading and depends on the nature of the porous material and the presence of an air layer between droplet and this porous surface.

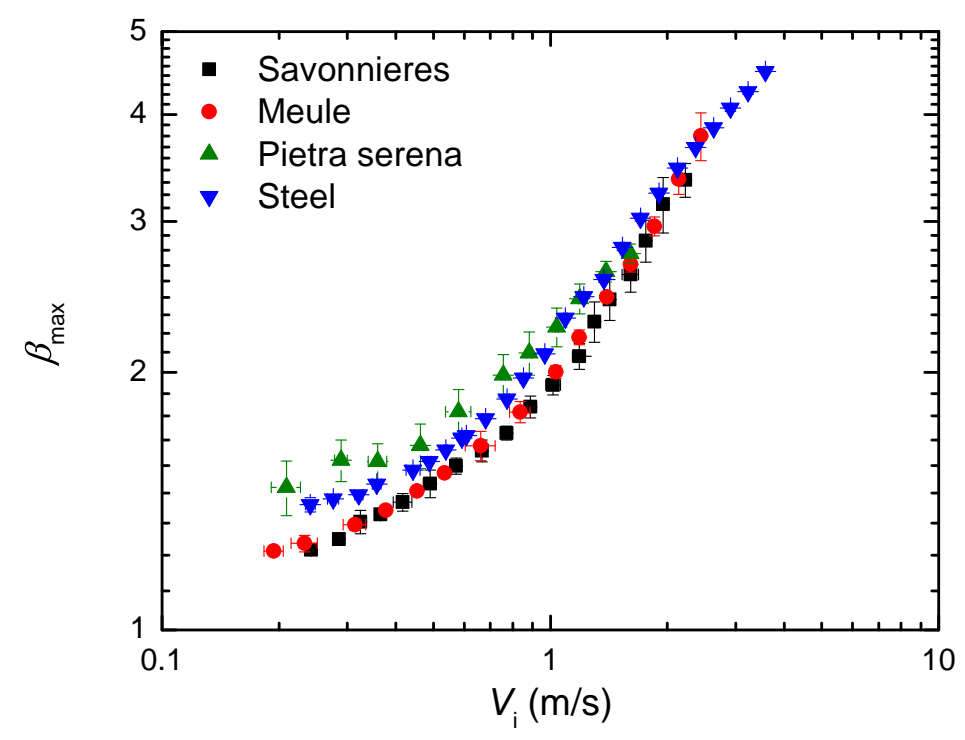

Fig. 6 Maximum spreading ratio as a function of impact velocity for drop impact on porous stones and steel in log-log plot.

We first analyze in more detail the first explanation. We recall that droplet spreading is a quite fast phenomenon reaching its maximum spreading in $1 \mathrm{E}-3$ to $1 \mathrm{E}-2 \mathrm{~s}$. Liquid penetration into the pore structure can be attributed to two phenomena: (1) at early time part of the volume of the droplet penetrates into the pore structure due to inertial effects at impact; (2) at later time, water is uptaken from the droplet due to capillary absorption by the substrate.

To study the inertial effect, we estimate the penetration depth $z$ and the time $\tau$ over which penetration occurs into the porous substrate. Consider a cylindrical volume of water that has entered a cylindrical pore, $\pi r R^{2} z$, to a depth $z$ with $R$ the radius. The momentum in this liquid is given by:

$$
P_{L}=\pi r R^{2} z \rho V_{\mathrm{i}}
$$

The penetrated liquid is acted upon by a frictional force due to viscous drag which tries to bring the liquid to a halt. The viscous force per unit area is: 


$$
f_{F}=\mu \frac{V_{i}}{R}
$$

This force works on area $A=2 \pi R z$ for time $\tau \approx z / V_{\mathrm{i}}$ producing the change in momentum as:

$$
P_{F}=2 \pi R z f_{F} \tau \approx 2 \pi R z \mu \frac{V_{\mathrm{i}}}{R} \frac{z}{V_{\mathrm{i}}} \approx 2 \pi z^{2} \mu
$$

Neglecting capillary penetration at this time scale, the penetration depth $z$ can then be found from equating $P_{L}$ and $P_{F}$ (respectively eqns. 3 and 5):

$$
z \approx V_{i} \frac{\rho R^{2}}{\mu}
$$

Considering an equivalent pore radius of $R_{\mathrm{eq}}=1 \mathrm{E}-5 \mathrm{~m}$ for e.g. Meule [16], an impact velocity $V_{\mathrm{i}}=2$ $\mathrm{m} / \mathrm{s}$ and initial droplet diameter $D_{0}=2.0 \mathrm{~mm}$, the penetration depth equals $z \approx 0.2 \mathrm{~mm}$ and time $\tau \approx$ $1 \mathrm{E}-4 \mathrm{~s}$. The time where penetration would occur is much shorter $\tau \approx 1 \mathrm{E}-4 \mathrm{~s}$ than the time it takes to reach maximum spreading $t \approx 1 \mathrm{E}-2 \mathrm{~s}$. We now analyze the mass in the porous media at these time scales.

Figure 7 shows the mass in the porous stone $M_{\mathrm{abs}}$, versus time in semi-log plot. The mass in the stone is determined using equation 2 . The remaining drop volume could only be measured accurately once the inertia forces are dissipated $(t>0.1 \mathrm{~s})$. Figures $7 \mathrm{a}-\mathrm{b}$ show that the main uptake process attributed to capillary absorption occurs for times $t>0.1 \mathrm{~s}$, thus after maximum spreading of the droplet. At early time $(t<0.01 \mathrm{~s})$, the liquid mass in the stone is negligible for Savonnières and Meule at impact velocities $<1 \mathrm{~m} / \mathrm{s}$. Extrapolating the curve for the Savonnières to the time at maximum spreading $(t \sim$ $0.01 \mathrm{~s}$ ) for the impact velocity of $2 \mathrm{~m} / \mathrm{s}$ shows also that liquid penetration is negligible. Extrapolating the curve to $1 \mathrm{E}-4 \mathrm{~s}$ the estimated time of penetration due to inertia at impact for Meule, at $V_{i}=2 \mathrm{~m} / \mathrm{s}$, also makes us conclude that no penetration due to inertial effects occurs. Also, Figure 6 shows that the maximum spreading ratio at $2 \mathrm{~m} / \mathrm{s}$ for Meule equals the maximum spreading of the impermeable steel surface, indicating once more no penetration occurred at maximum spreading.

We conclude that, for the porous stones studied, the volume loss of the droplet by liquid penetration into the porous substrates due to inertial effects at impact can be neglected and that mass uptake by the substrate due to capillary absorption only starts after maximum spreading of the droplet. This is explained by the existence of above mentioned air layer, which is entrained between the droplet and the porous substrate during the spreading process preventing penetration either due to inertial effects or capillary absorption. 


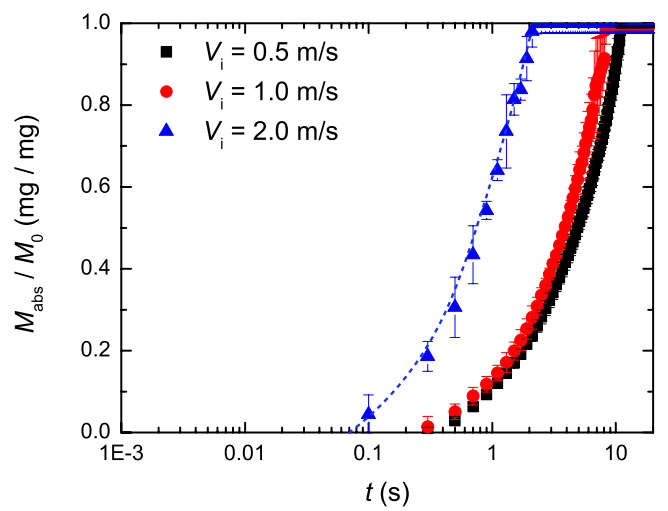

(a)

Figure 7: Normalized penetration mass into the pore versus time: (a) Savonnières and (b) Meule for different impact velocities $V_{\mathrm{i}}=0.5,1.0$ and $2.0 \mathrm{~m} / \mathrm{s}$.

To analyze assumption (2) on the influence of dynamic wetting, we study the maximum spreading ratio on porous and impermeable surfaces in more detail using the energy balance approach. Maximum spreading is governed by the balance between kinetic energy, capillary or surface tension energy before impact and at maximum spreading in surplus of the viscous dissipation during spreading. Energy balance models are commonly formulated using two dimensionless parameters: the Weber number ( $\mathrm{We}=\rho D_{0} V_{\mathrm{i}}^{2} / \gamma$ ) describing the ratio between the kinetic and capillary energy and the Reynolds number $\left(\operatorname{Re}=\rho D_{0} V_{\mathrm{i}} / \mu\right)$ describing the ratio between the kinetic and viscous energy. Based on energy balance at low and high impact velocity, two common limit regimes have been introduced: the capillary regime at low impact velocity and the viscous regime at high impact velocity [9]. Clanet et al. [9] proposed, based on momentum conservation, a scaling of $\beta_{\max } \sim \mathrm{We}^{1 / 4}$. Based on energy conservation between kinetic and surface energy, a scaling of $\beta_{\max } \sim \mathrm{We}^{1 / 2}$ is found $[25,26]$. Figure $8 \mathrm{a}$ compares the measured maximum spreading ratio and the two scaling models. No agreement with the scaling of $\beta_{\max } \sim \mathrm{We}^{1 / 2}$ is observed, especially at low impact velocity. Also the scaling with $\mathrm{We}^{1 / 4}$ is found not to be consistent especially for Savonnières and Meule at low impact velocity. To analyze the scaling of $\beta_{\max }$ with $\mathrm{We}^{1 / 4}$ in more detail, we performed additional drop impact experiments on Savonnières with two other liquids: 1:1.3 glycerol-water mixture (glycerol $10 \mathrm{mPa} \cdot \mathrm{s}$ ) and pure ethanol. Glycerol has a ten times lower viscosity $(1.2 \mathrm{E}-3 \mathrm{~Pa} \cdot \mathrm{s})$ than water, while ethanol has a three times lower surface tension $(2.3 \mathrm{E}-2 \mathrm{~N} / \mathrm{m})$. Figure $8 \mathrm{~b}$ clearly shows that the $\mathrm{We}^{1 / 4}$ scaling does not apply to our data. In general, the scaling of $\beta_{\max } \sim \mathrm{We}^{\alpha}$ implies that, at zero impact velocity, $\beta_{\max }$ the maximum spreading ratio equals zero, which is physically impossible $\left(\beta_{\max } \geq 1\right)$. Figure 8 a reveals that, at low impact velocity, the spreading ratio does not tend to zero, but levels off to a constant maximum spreading ratio.

In the viscous regime at high impact velocity, based on energy conservation between kinetic and viscous dissipation energy, a scaling of $\beta_{\max }$ with $\operatorname{Re}^{1 / 5}$ is found $[9,27,28]$. Figure 8 c compares the 
measured maximum spreading ratio and scaling of $\beta_{\max }$ with $\mathrm{Re}^{1 / 5}$. We can observe that data for impact on impermeable and porous materials converge to a single curve, although it would be challenging to conclude that the data tend to a slope of $1 / 5$. Our data do not cover sufficiently high impact velocity to evaluate properly the scaling in the viscous regime, since splashing occurs at such higher impact velocity.

An universal scaling of the maximum spreading with broad cross over from capillary to viscous regime has been proposed by Laan et al. [29] for impermeable surfaces by interpolating between two scaling models, i.e. $\mathrm{We}^{1 / 2}$ and $\mathrm{Re}^{1 / 5}$. Figure $8 \mathrm{~d}$ shows the rescaling as function of the impact number $\left(P=\mathrm{We} \cdot \mathrm{Re}^{-2 / 5}\right)$ for all substrates. At high $P$, one may conclude that a convergence of the curves as predicted by the model of Laan is observed. As $P$ becomes smaller, the data deviate from the predicted curve and, even at very low $P$, the data start to increase again. These observations show that the existing scaling laws do not predict our data correctly. When taking into account the dynamic wetting behavior correctly as suggested by Lee et al.[23], the rescaled data collapse into a single curve, as shown below.
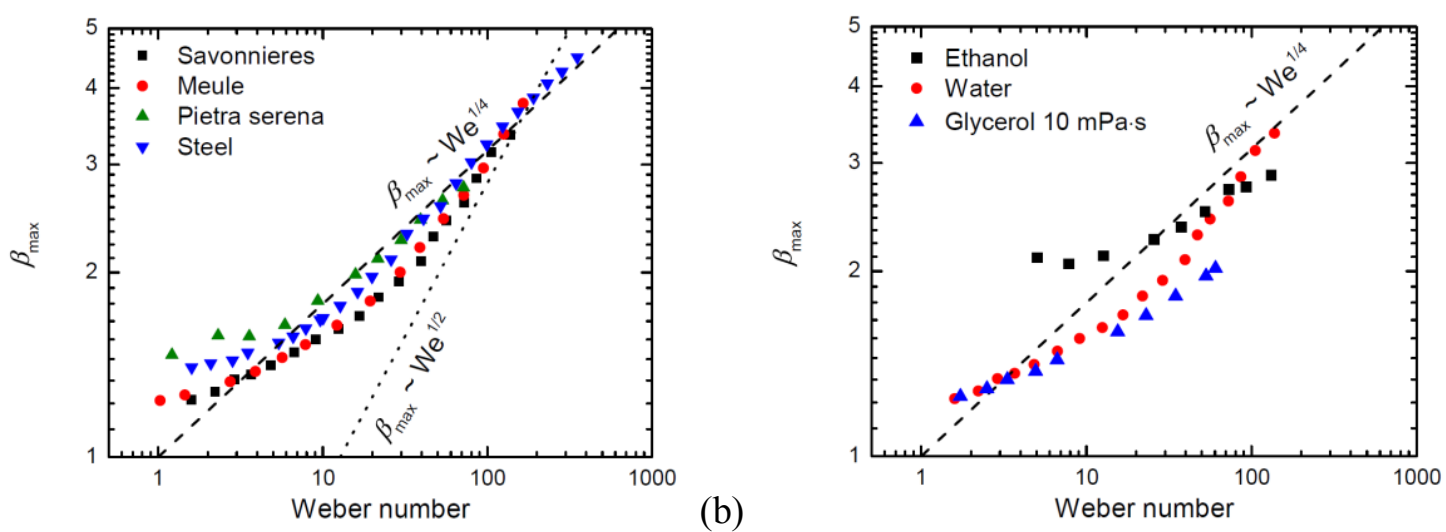

(a)

(b)

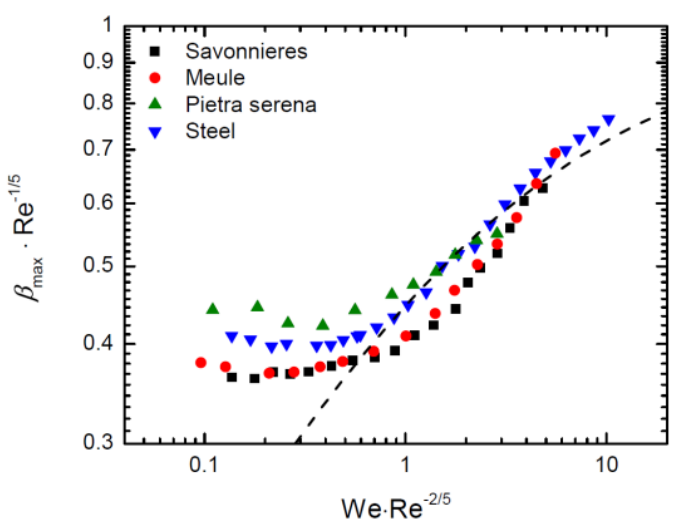

(c)

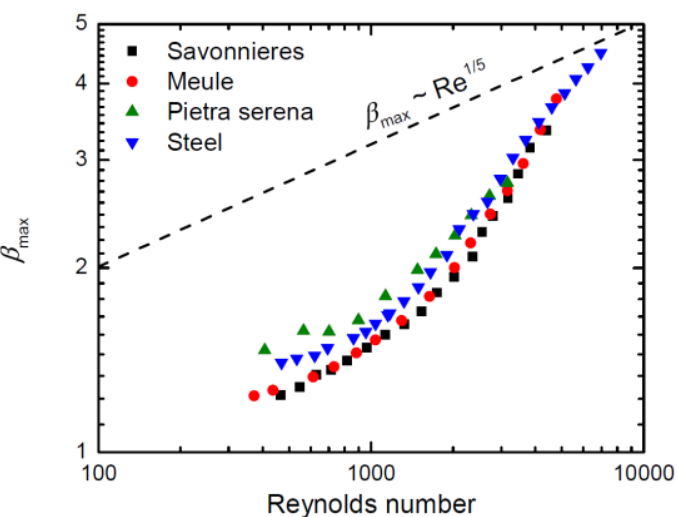

(d)

$\mathrm{We} \cdot \operatorname{Re}^{-2 / 5}$

Fig. 8 Maximum spreading ratio as function of Weber number with scaling laws $\beta_{\max } \sim \mathrm{We}^{1 / 4}$ and $\beta$ $\max _{\max } \sim \mathrm{We}^{1 / 2}$ (a) for water on different substrates; (b) for glycerol, water and ethanol on Savonnières. (c) Maximum spreading ratio as function of Reynolds number and scaling with $\mathrm{Re}^{1 / 5}$. (d) Rescaled 
spreading ratio as function of the impact number $P=\mathrm{We} \cdot \mathrm{Re}^{-2 / 5}$ for water impact on different substrates.

To account for the dynamic wetting behavior, we add a term to the energy balance between kinetic energy before impact $E_{\mathrm{k}}$ and capillary energy at maximum spreading $E_{\gamma}$, by taking into account the capillary or surface tension energy at the low impact velocity limit $E_{\gamma 0}$. The energy balance then reads:

$$
E_{k}+E_{\gamma 0} \sim E_{\gamma}
$$

or

which leads to

$$
\rho D_{0} V_{i}^{2}+\gamma D_{V i=0}^{2}=\gamma D_{\max }^{2}
$$

$$
\sqrt{\beta_{\max }^{2}-\beta_{V i=0}^{2}}=\mathrm{We}^{1 / 2}
$$

where $D_{\mathrm{Vi}=0}=\beta_{\mathrm{Vi}=0} \cdot D_{0}$. Equation 9 shows that the data after correction for $\beta_{\mathrm{Vi}=0}$ should scale with $\mathrm{We}^{1 / 2}$.

To determine the spreading ratio $\beta_{\mathrm{Vi}=0}$ the measured data are approximated by the following function:

$$
\beta_{\text {max }}=\beta_{V \mathrm{i}=0}+A \cdot \frac{V_{\mathrm{i}}^{C}}{B+V_{\mathrm{i}}^{C}}
$$

where $A, B, C$ and $\beta_{\mathrm{Vi}=0}$ are obtained by fitting the curve to our data. Figure 9 shows that eqn. 10 fits the data satisfactorily allowing to determine the spreading ratio $\beta_{\mathrm{Vi}=0}$. The values for $\beta_{\mathrm{Vi}=0}$ are given in the inset of the figure. We observe that the spreading ratios $\beta_{\mathrm{Vi}=0}$ for Savonnières and Meule, showing a higher surface porosity, are lower than for the impermeable substrate, while the $\beta_{\mathrm{Vi}=0}$ for Pietra Serena is higher. This observation is consistent with the porosity values, but the higher $\beta_{\mathrm{Vi}}=$ for Pietra Serena compared to the non-porous steel surface has still to be explained further.

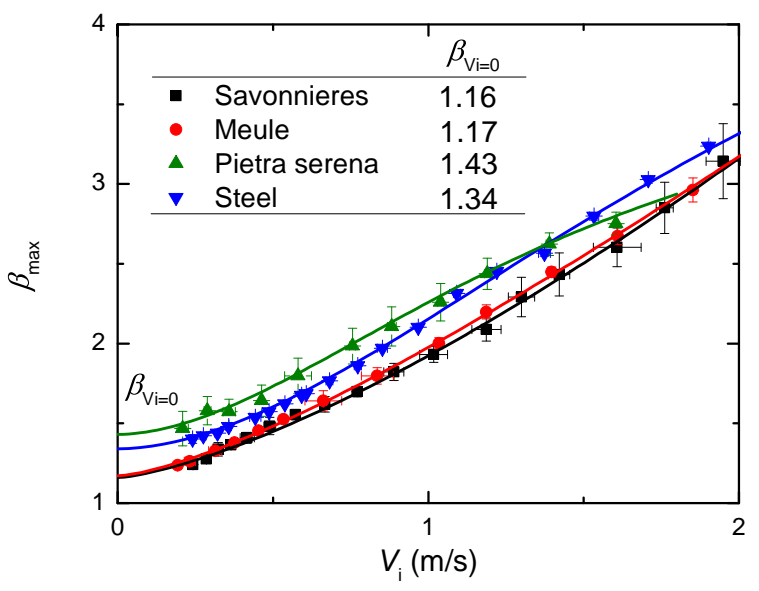

Fig. 9. Determination of the maximum spreading ratio at zero impact velocity by fitting eqn. 4 to the measured data. 
According to eqn. 9, the corrected maximum spreading $\left(\beta_{\max }^{2}-\beta_{V i=0}^{2}\right)^{1 / 2}$ should scale with $\mathrm{We}^{1 / 2}$. Therefore we can still apply the approach of Laan et al [29] for the corrected maximum spreading ratio, where a smooth transition between the capillary and viscous regimes is described as:

$$
\left(\beta_{\text {max }}^{2}-\beta_{V i=0}^{2}\right)^{1 / 2} \cdot \mathrm{Re}^{-1 / 5}=\mathrm{We}^{1 / 2} /\left(A+\mathrm{We}^{1 / 2}\right)
$$

which is based on the first order Padé approximation. Figure 10 shows the corrected measured data $\left(\beta_{\max }^{2}-\beta_{V i=0}^{2}\right)^{1 / 2}$ as a function of Weber number for drop impact on porous stones and steel surface. The curve as predicted by eqn. 11 , with $\mathrm{A}=7.6$, shows a good agreement with the rescaled data for maximum spreading. The larger error bars at low velocity are due to the logarithmic scaling. This result shows that we can rescale the maximum spreading ratio for different porous media and for impermeable substrate into a single description, when the data are corrected for the limiting spreading ratio at zero velocity $\beta_{\mathrm{Vi}=0}$.

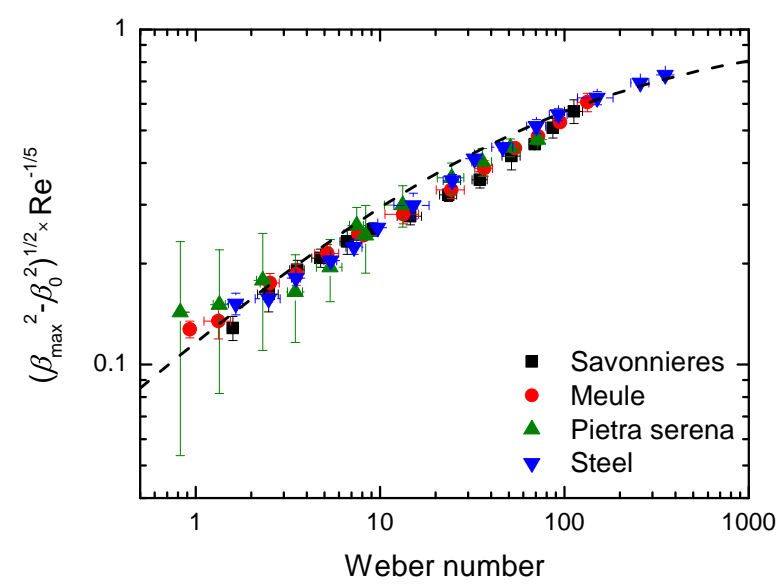

Fig. 10 Rescaled maximum spreading ratio as a function of Weber number in log-log plot for porous stones. The dotted line is the first order Padé approximation (Eqn. 9).

Finally, we try to unravel the physical meaning of the limiting spreading ratio at zero velocity $\beta_{\mathrm{Vi}=0}$. For this we explore the energy balance for a pancake-shaped droplet at maximum spreading, including kinetic and surface energy before impact, and surface energy and viscous dissipation at maximum spreading [30], given by:

$$
(\mathrm{We}+12) \beta_{\text {max }}=8+\beta_{\text {max }}^{3}\left[3(1-\cos \theta)+\frac{4 W e}{\sqrt{R e}}\right]
$$

To find an expression for $\beta_{V_{i} \rightarrow 0}$, the limit for $V_{i} \rightarrow 0$ is taken in eqn. 12: 


$$
\beta_{V_{i} \rightarrow 0}{ }^{2}=\frac{4}{\left(1-\cos \theta_{V i=0}\right)}\left(1-\frac{2}{3 \beta_{V_{i} \rightarrow 0}}\right)
$$

This equation shows that the limiting spreading ratio depends on a contact angle, which we denote as $\theta_{\mathrm{Vi}=0}$, its value can be obtained directly from $\beta_{\mathrm{Vi}=0}$ using eq. 13 . The dynamic wetting behavior at maximum spreading is characterized by the dynamic contact angle $\theta_{\mathrm{D}}\left(t_{\max }\right)$ imaged and measured at maximum spreading time $t_{\max }$. Figure $11 \mathrm{a}$ compares the contact angle $\theta_{\mathrm{Vi}=0}$ determined from $\beta_{\mathrm{Vi}=0}$ using eqn. 13 and the dynamic contact angle $\theta_{\mathrm{D}}\left(t_{\max }\right)$ at maximum spreading, showing a reasonable agreement. This means that the limiting spreading ratio $\beta_{\mathrm{Vi}=0}$ describes the dynamic wetting behavior during wetting and is characterized by the dynamic contact angle $\theta_{\mathrm{D}}\left(t_{\max }\right)$.

The derivation of this equation is based on the assumption that the substrate is non-porous or $\Phi$ is zero. Assuming however that the droplet is in the Cassie-Baxter state on a porous substrate, the surface tension energy at maximum spreading reads $\mathrm{SE}_{2}=S_{1} \cdot \gamma_{\mathrm{LV}}+S_{2} \cdot\left(\gamma_{\mathrm{SL}}-\gamma_{\mathrm{SV}}\right)(1-\Phi)+S_{2}$. $\gamma_{\mathrm{LV}} \Phi$, where $S_{1}$ is the surface between droplet and surrounding air and $S_{2}$ is the contact area droplet substrate. This leads to a modification of Eq. 13 for $\beta_{V_{i} \rightarrow 0}$ :

$$
\beta_{\max }^{2}=\frac{4}{(1+\Phi)-(1-\Phi) \cos \theta}\left(1-\frac{2}{3 \beta_{\max }}\right)
$$

The comparison of the contact angles obtained from this relation with our data of dynamic contact at maximum spreading does not give a better agreement (Fig. 11b). This may indicate that a direct relation between (surface) porosity and dynamic contact does not apply, and other aspects have to be considered.
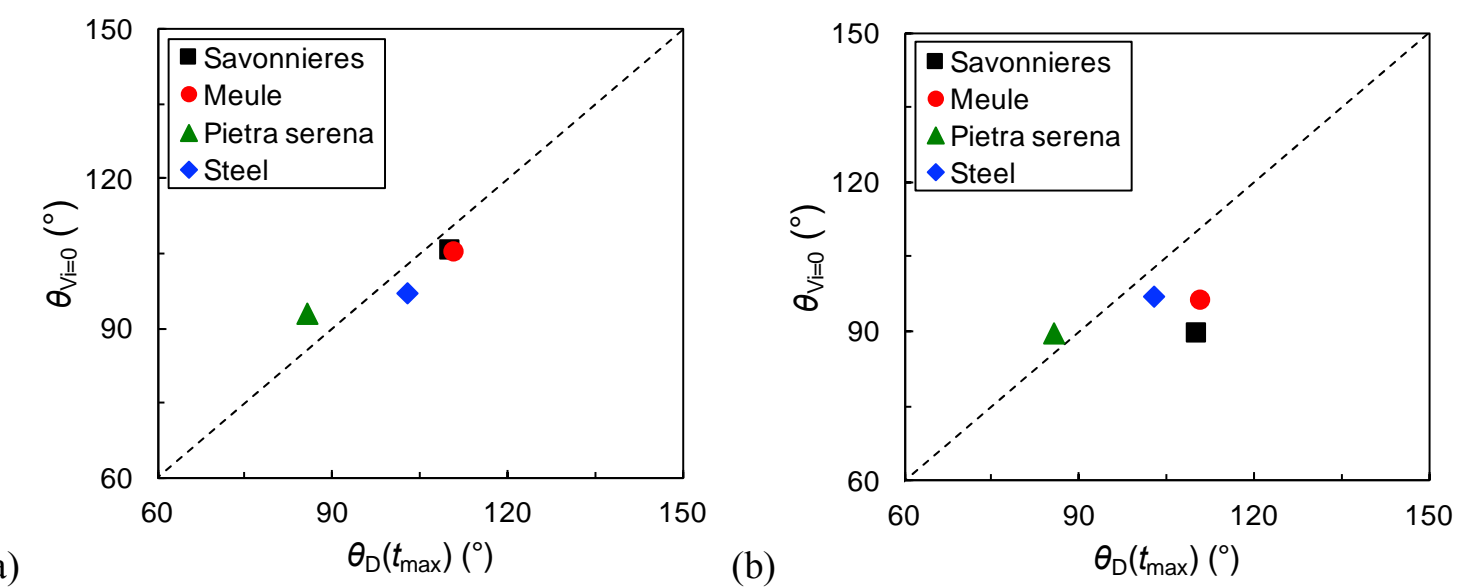

Fig. 11 (a) Comparison of the dynamic contact angle $\theta_{\mathrm{D}}\left(t_{\max }\right)$ (from experiments) and the contact angle $\theta_{\mathrm{Vi}=0}$ determined from $\beta_{\mathrm{Vi}=0}$ using eqn. 13 assuming no porosity. (b) Comparison of the dynamic contact angle $\theta_{\mathrm{D}}\left(t_{\max }\right)$ (from experiments) and the contact angle $\theta_{\mathrm{Vi}=0}$ determined from $\beta_{\mathrm{Vi}=0}$ using eqn. 13 assuming surface porosity. 
It is shown that the maximum spreading can be rescaled properly for porous and impermeable surfaces by taking the capillary energy at zero impact velocity into account. We hypothesize that the origin of this successful rescaling for porous stone can be attributed to the presence of a thin air layer between droplet and the porous stone during spreading, which leads to a similar spreading behavior on Savonnières and Meule. The different behavior of Pietra Serena, showing a lower dynamic contact at low impact velocity and a higher spreading ratio $\beta_{\mathrm{Vi}=0}$ than an impermeable surface, is possibly caused by the (partially) breaking of this air layer, leading to a dynamic contact angle $<90^{\circ}$ causing capillary forces to enhance the dynamic wetting of the surface.

This analysis demonstrates that dynamic wetting plays an important role in the spreading at low velocity and that the dynamic wetting as characterized by the dynamic contact angle $\theta_{\mathrm{D}}$ has to be taken into account for predicting the maximum spreading. The analysis above shows that the capillary or surface tension energy related to $\theta_{\mathrm{Vi}=0}$ or to $\beta_{\mathrm{Vi}=0}$ has to be incorporated in the prediction of the maximum spreading. The relation between the dynamic contact angle at maximum spreading, the dynamic contact angle $\theta_{\mathrm{Vi}=0}$ and maximum spreading ratio $\beta_{\mathrm{Vi}=0}$ at zero velocity, and its connection to surface properties such as surface porosity and roughness remains however still unclear.

\section{Conclusions}

Drop impact on porous stones has been experimentally investigated by high-speed imaging and compared to the drop impact on impermeable surface. For the porous stones studied, no liquid penetrated into the porous substrate due to inertial effects at impact, and the liquid in the porous substrate is only due to capillary absorption by the porous substrate starting after maximum spreading of the droplet. At maximum spreading the contact line on porous substrates pins and the area at maximum spreading determines the contact area between fluid and porous stones for capillary uptake. The dynamic non-wetting behavior during spreading and hydrophobic contact angle $>90^{\circ}$ is attributed to the presence of an air layer between droplet and porous substrate. The maximum dynamic contact angle is found to increase for porous stones with higher porosity. The droplet gets pinned when the air layer is broken at the contact line and capillary forces develop in fines pores at the droplet edge pinning the droplet. This pinning due capillary absorption at the droplet edge leads to a change from dynamic non-wetting to a wetting behavior with a contact angle $<90^{\circ}$. Air remains entrapped under the pinned droplet.

Maximum spreading on porous stones increases with impact velocity but does not scale with Weber number at low impact velocity. It is demonstrated that dynamic wetting plays an important role in the spreading at low velocity and that the dynamic wetting as characterized by the dynamic contact angle $\theta_{\mathrm{D}}$ has to be taken into account for predicting the maximum spreading. Correcting the maximum spreading ratio for the dynamic wetting behavior, it is demonstrated that all data for porous stones and 
non-porous substrates collapse onto a single curve. The relation between the dynamic contact angle at maximum spreading, the dynamic contact angle $\theta_{\mathrm{Vi}=0}$ and maximum spreading ratio $\beta_{\mathrm{Vi}=0}$ at zero velocity, and its connection to surface properties such as surface porosity and roughness remains however unclear.

The results of this study are relevant for applications where the mass transfer between the impacting droplet and the porous medium has to be understood. Particularly the initial phase of the absorption process where a resistance seems to appear has to be further investigated.

\section{Acknowledgements}

The authors acknowledge the support of Swiss National Science Foundation grant no. 200021_135510.

\section{Appendix A. Supplementary material}

Supplementary data associated with this article can be found, in the online version, at XXXXXX.

\section{Reference}

[1] A. Clarke, T.D. Blake, K. Carruthers, A. Woodward, Spreading and Imbibition of Liquid Droplets on Porous Surfaces, Langmuir 18 (2002) 2980-2984.

[2] K. Wallace, K. Yoshida, Determination of dynamic spread factor of water droplets impacting on water-sensitive paper surfaces, Journal of Colloid and Interface Science. 63 (1978) 164-165.

[3] S. Chandra, C. Avedisian, Observations of droplet impingement on a ceramic porous surface, International Journal of Heat and Mass Transfer. 35 (1992) 2377-2388.

[4] Z. Yu, F. Wang, L.S. Fan, Experimental and numerical studies of water droplet impact on a porous surface in the film-boiling regime, Industrial and Engineering Chemistry Research. 47 (2008) 9174-9182.

[5] N.C. Reis Jr., R.F. Griffiths, J.M. Santos, Numerical simulation of the impact of liquid droplets on porous surfaces, Journal of Computational Physics. 198 (2004) 747-770.

[6] N.C. Reis Jr., R.F. Griffiths, J.M. Santos, Parametric study of liquid droplets impinging on porous surfaces, Applied Mathematical Modelling. 32 (2008) 341-361.

[7] J.O. Marston, S.T. Thoroddsen, W.K. Ng, R.B.H. Tan, Experimental study of liquid drop impact onto a powder surface, Powder Technology. 203 (2010) 223-236.

[8] H. Katsuragi, Length and time scales of a liquid drop impact and penetration into a granular layer, J. Fluid Mech. 675 (2011) 552-573.

[9] C. Clanet, C. Béguin, D. Richard, D. Quéré, Maximal deformation of an impacting drop, J. Fluid Mech. 517 (2004) 199-208.

[10] G. Delon, D. Terwagne, S. Dorbolo, N. Vandewalle, H. Caps, Impact of liquid droplets on granular media, Phys. Rev. E. 84 (2011) 046320.

[11] E. Nefzaoui, O. Skurtys, Impact of a liquid drop on a granular medium: Inertia, viscosity and surface tension effects on the drop deformation, Experimental Thermal and Fluid Science. 41 (2012) 43-50. 
[12] R. Zhao, Q. Zhang, H. Tjugito, X. Cheng, Granular impact cratering by liquid drops: Understanding raindrop imprints through an analogy to asteroid strikes, Proceedings of the National Academy of Sciences. 112 (2015) 342-347.

[13] S.-C. Zhao, R. de Jong, D. van der Meer, Raindrop impact on sand: a dynamic explanation of crater morphologies, Soft Matter. 11 (2015) 6562-6568.

[14] H. Derluyn, Salt Transport and Crystallization in Porous Limestone, PhD Thesis, ETH Zurich, 2012.

[15] R. Dreesen, M. Dusar, Historical building stones in the province of Limburg (NE Belgium): role of petrography in provenance and durability assessment, Materials Characterization. 53 (2004) 273-287.

[16] P. Moonen, Contiuous-discontinuous modelling of hygrothermal damage processes in porous media, PhD Thesis, TU Delft, Delft University of Technology, 2009.

[17] S. Roels, J. Carmeliet, H. Hens, Modelling Unsaturated Moisture Transport in Heterogeneous Limestone, Transp Porous Med. 52 (2003) 333-350.

[18] L. Roels, Modelling unsaturated moisture transport, PhD Thesis, KU Leuven, 2000.

[19] H. Ardebrant, R.J. Pugh, Wetting studies on silicate minerals and rocks used in bituminous highways, Colloids and Surfaces. 58 (1991) 111-130.

[20] T. Okayama, D.S. Keller, P. Luner, The wetting of calcite surfaces, The Journal of Adhesion. 63 (1997) 231-252.

[21] M. Rodriguez-Valverde, M. Cabrerizo-Vilchez, P. Rosales-Lopez, A. Paez-Duenas, R. Hidalgo-Alvarez, Contact angle measurements on two (wood and stone) non-ideal surfaces, Colloids and Surfaces a: Physicochemical and Engineering Aspects. 206 (2002) 485-495.

[22] D. Biolè, V. Bertola, A goniometric mask to measure contact angles from digital images of liquid drops, Colloids and Surfaces a: Physicochemical and Engineering Aspects. 467 (2015) 149-156.

[23] J.B. Lee, N. Laan, K.G. de Bruin, G. Skantzaris, N. Shahidzadeh, D. Derome, et al., Universal rescaling of drop impact on smooth and rough surfaces, J. Fluid Mech. 786 (2016) R4.

[24] F. Dullien, Porous Media Fluid Transport and Pore Structure, Elsevier, 2012.

[25] E.W. Collings, A.J. Markworth, J.K. McCoy, J.H. Saunders, Splat-quench solidification of freely falling liquid-metal drops by impact on a planar substrate, Journal of Materials Science. 25 (1990) 3677-3682.

[26] T. Bennett, D. Poulikakos, Splat-quench solidification: estimating the maximum spreading of a droplet impacting a solid surface, Journal of Materials Science. 28 (1993) 963-970.

[27] J. Madejski, Solidification of droplets on a cold surface, International Journal of Heat and Mass Transfer. 19 (1976) 1009-1013.

[28] I.V. Roisman, R. Rioboo, C. Tropea, Normal impact of a liquid drop on a dry surface: model for spreading and receding, Proceedings of the Royal Society of London a: Mathematical, Physical and Engineering Sciences. 458 (2002) 1411-1430.

[29] N. Laan, K.G. de Bruin, D. Bartolo, C. Josserand, D. Bonn, Maximum Diameter of Impacting Liquid Droplets, Phys. Rev. Applied. 2 (2014) 044018.

[30] T. Mao, D. Kuhn, H. Tran, Spread and rebound of liquid droplets upon impact on flat surfaces, AIChE J. 43 (1997) 2169-2179. 